\title{
Estimation of the Reproduction Number of Influenza A(H1N1)pdm2009 in South Korea Using Heterogeneous Models
}

\section{Yunjeong Lee}

Yonsei University

\section{Dong Han Lee}

Korea Centers for Disease Control and Prevention

Hee-Dae Kwon

Inha University

Changsoo Kim

Yonsei University College of Medicine

jeehyun lee ( $\nabla$ ezhyun71@yonsei.ac.kr)

Yonsei University

Research article

Keywords: Influenza, Reproduction number, Antiviral agents

Posted Date: October 21st, 2020

DOI: https://doi.org/10.21203/rs.3.rs-20387/v3

License: (c) (1) This work is licensed under a Creative Commons Attribution 4.0 International License.

Read Full License

Version of Record: A version of this preprint was published at BMC Infectious Diseases on July 7th, 2021.

See the published version at https://doi.org/10.1186/s12879-021-06121-8. 
Yunjeong Lee ${ }^{1 \dagger}$, Dong Har Lee ${ }^{2 \dagger}$, Hee-Dae Kwon ${ }^{3}$, Changsoo Kim ${ }^{4}$ and Jeehyun Lee ${ }^{*} \quad 9$

${ }^{*}$ Correspondence:

ezhyun@yonsei.ac.kr

${ }^{5}$ Department of Mathematics,

Yonsei University, 50, Yonsei-ro, 03722, Seoul, South Korea

Full list of author information is available at the end of the article

$\dagger$ Equal contributor
10

Keywords: Influenza; Reproduction number; Antiviral agents

\section{Abstract} differences illustrated in the incidence data. the robust estimation of parameters.
Background: The reproduction number is one of the most crucial parameters in determining disease dynamics, providing a summary measure of the transmission potential. However, estimating this value is particularly challenging owing to the characteristics of epidemic data, including non-reproducibility and incompleteness.

Results: In this study, we propose mathematical models with different population structures; each of these models can produce data on the number of cases of the influenza $A(H 1 N 1) p d m 09$ epidemic in South Korea. These structured models incorporating the heterogeneity of age and region are used to estimate the reproduction numbers at various terminal times. Subsequently, the age- and region-specific reproduction numbers are also computed to analyze the

Conclusions: Numerical results reveal that the introduction of heterogeneity into the population to represent the general characteristics of dynamics is essential for

26

27

\section{${ }^{28}$ Background}

${ }^{29}$ The reproduction number is defined as the average number of secondary cases gen- ${ }^{29}$ 30 erated by a typical primary case. It is a measure of the transmission potential asso- 30 ${ }^{31}$ ciated with the contact rate, duration of infectivity, and probability of transmission ${ }^{31}$ ${ }^{32}$ per contact. The maximum reproduction number is attained when an infectious ${ }^{32}$ ${ }^{33}$ person is introduced into a totally susceptible population and is called the basic ${ }^{33}$ 
${ }^{1}$ reproduction number, $R_{0}$. Various approaches such as the exponential growth rate ${ }^{1}$

${ }^{2}$ of infections during the early epidemic stage, model-based schemes, and maximum- ${ }^{2}$

${ }^{3}$ likelihood estimations have been used to analyze this number [1-4]. ${ }^{3}$

4 When an infection spreads throughout a population, the time-dependent effective ${ }^{4}$

${ }^{5}$ reproduction number, $R_{t}$, is often more useful for assessing the transmission po- $^{5}$

${ }^{6}$ tential throughout a pandemic, especially during the period with the highest level ${ }^{6}$

${ }^{7}$ of activity. Real-time estimation continues to track the number of secondary in- ${ }^{7}$

${ }^{8}$ fections caused by a single infective, providing a quantitative measure of the time ${ }^{8}$

${ }^{9}$ evolution of the epidemic force. Cruz-Pacheco et al. demonstrated the manner in ${ }^{9}$

${ }^{10}$ which sanitary measures reduce the prevalence of an infected population [1]. Esti- ${ }^{10}$

${ }^{11}$ mates of the reproduction number were shown to decrease from 1.4-1.5 initially to ${ }^{11}$

${ }^{12} 1.1-1.2$ later in the summer, which was most likely because of the vacation period ${ }^{12}$

${ }^{13}$ and the seasonality of influenza transmission [5]. In addition to capturing temporal ${ }^{13}$

${ }^{14}$ dynamics, it is important to consider heterogeneous patterns of the transmission. ${ }^{14}$

${ }^{15}$ It is well known that school-age children are disproportionately responsible for in- ${ }^{15}$

${ }^{16}$ fluenza transmissions. Estimates of the age-specific reproduction number help with ${ }^{16}$

${ }^{17}$ our understanding of the role of each group in the transmission dynamics and with ${ }^{17}$

${ }^{18}$ devising effective targeting mitigation strategies. 18

19 If all incident cases could be traced back to their index cases, estimating the ${ }^{19}$

${ }^{20}$ reproduction number would simply be a matter of counting the number of secondary ${ }^{20}$

${ }^{21}$ cases. However, with most epidemics, only the epidemic curve is observed, and ${ }^{21}$

${ }^{22}$ there is no available information regarding who infected whom. To appropriately ${ }^{22}$

${ }^{23}$ estimate the reproduction number from the influenza outbreak data, it is essential ${ }^{23}$

${ }^{24}$ that the selected model capture the underlying dynamics embedded in the data. ${ }^{24}$

${ }^{25}$ The objective of this study is to estimate the reproduction numbers based on the ${ }^{25}$ ${ }^{26}$ incidence data.

${ }^{27}$ When the World Health Organization announced the emergence of influenza ${ }^{27}$

${ }^{28} \mathrm{~A}(\mathrm{H} 1 \mathrm{~N} 1)$ pdm09 (pH1N1) in 2009 [6], the first probable patient in South Korea ${ }^{28}$

${ }^{29}$ was identified on April 28. A total of 763,759 confirmed cases, of which 270 were $^{29}$

${ }^{30}$ fatal, were reported by the end of August 2010 [7]. During the initial epidemic ${ }^{30}$

${ }^{31}$ phase, the main control measure was containment through quarantine and isola- ${ }^{31}$

${ }^{32}$ tion. Surveillance programs in schools and medical facilities were implemented, and ${ }^{32}$

${ }^{33}$ all confirmed cases were investigated. However, when community outbreaks were ${ }^{33}$ 
${ }^{1}$ detected in June, the intervention policy switched from containment to mitigation, ${ }^{1}$

${ }^{2}$ including vaccination and antiviral prescription. Vaccination was started on Octo- ${ }^{2}$

${ }^{3}$ ber 27, 2009, and 12.7 million people were vaccinated by the end of August 2010. ${ }^{3}$

${ }^{4}$ Before August 20, antiviral agents were prescribed to patients with acute febrile ${ }^{4}$

${ }^{5}$ respiratory illness (AFRI) and who had a history of travel abroad or contact with ${ }^{5}$

${ }^{6}$ a confirmed patient. However, when the number of community-acquired cases in- ${ }^{6}$

${ }^{7}$ creased, antiviral agents were prescribed to patients with AFRI symptoms. ${ }^{7}$

8 According to the database, $3,087,788$ courses of antiviral agents were prescribed ${ }^{8}$

${ }^{9}$ from August 21, 2009 to April 30, 2010. The daily number of incident patients ${ }^{9}$

${ }^{10}$ was estimated based on the amount of prescribed antiviral agents (Refer to [8] for ${ }^{10}$

${ }^{11}$ details). Figure 1 shows the temporal incidence distribution of pH1N1 in South ${ }^{11}$

${ }^{12}$. 12

Korea. The amount of antiviral agents prescribed and the number of incident pa-

${ }^{13}$ tients soared from mid-October, reached its peak at the end of October, and started ${ }^{13}$

${ }^{14}$ declining in mid-November.

Demographic and regional characteristics are illustrated in Table 1 and Figure

2. The incidence rate is higher in children and students than in other age group ${ }_{17}$

individuals (Table 1). The rate is higher in urban areas than in rural areas and is 18

the highest in the national capital and the south-eastern region (Figure 2).

In this paper, two different structured models are proposed to estimate reproduc- ${ }_{20}$ ${ }_{21}$ tion numbers on the basis of the epidemic curve. We begin by introducing a basic ${ }_{21}$ ${ }_{22}$ SIR model to describe a single outbreak and build age- and region-structured mod- ${ }_{22}$ ${ }_{23}$ els by incorporating population heterogeneity. Numerical simulations are conducted ${ }_{23}$ ${ }_{24}$ to analyze the impact of terminal time and the effect of heterogeneous structures ${ }_{24}$ ${ }_{25}$ on the estimation of parameters. Finally, the proposed models are applied to the ${ }_{25}$ ${ }_{26}^{2009}$ incidence data of novel pH1N1 in South Korea to compute the age- and region- ${ }_{26}$ ${ }_{27}$ specific reproduction numbers.

${ }^{31}$ We consider the standard SIR model to represent single-outbreak influenza dy- ${ }^{31}$ ${ }^{32}$ namics. The model classifies individuals into three key compartments: susceptible, ${ }^{32}$

${ }^{33}$ infected, and recovered. The nonlinear system of differential equations describing ${ }^{33}$ 


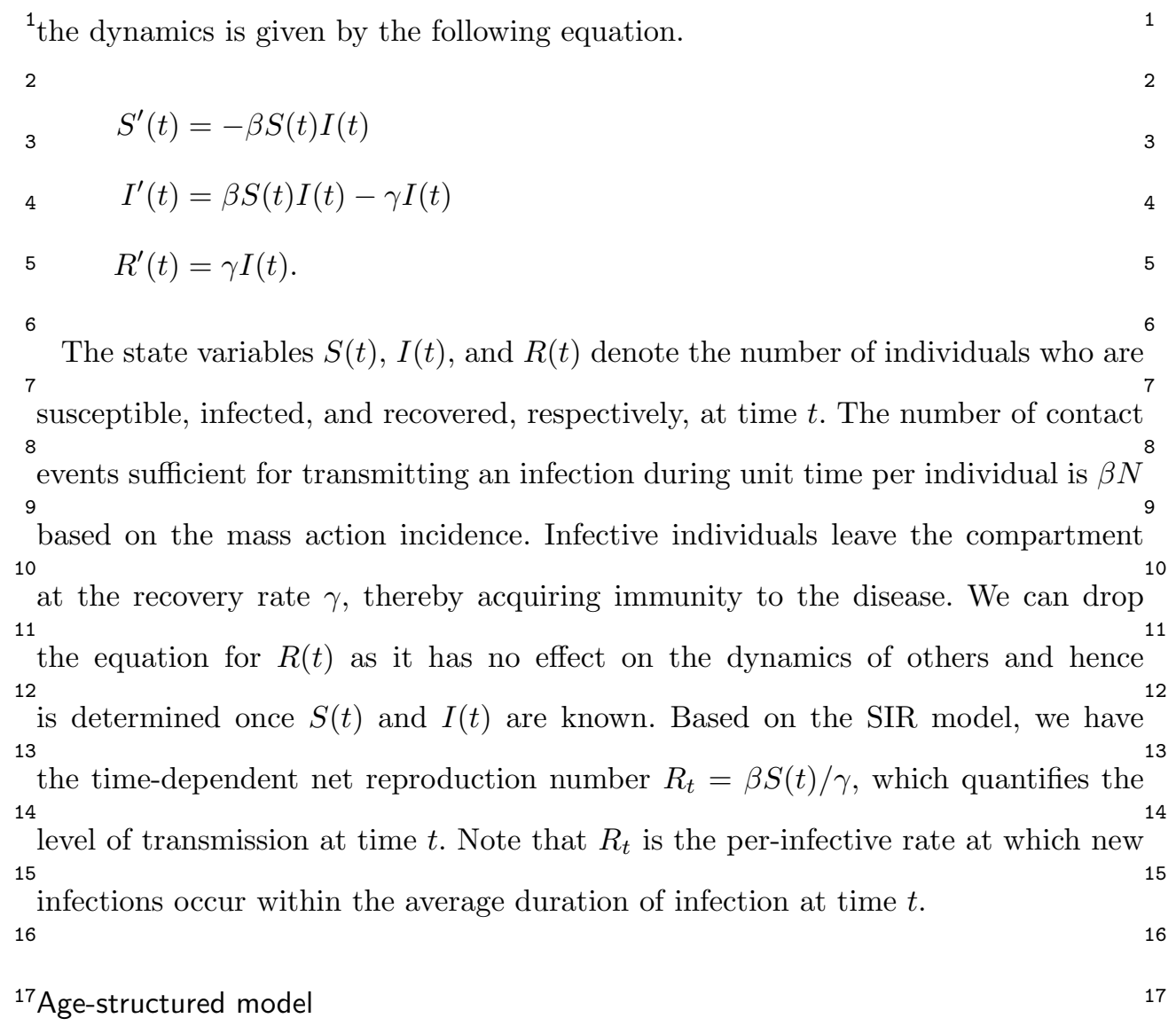

${ }^{18} \mathrm{An}$ age-structured model is employed to estimate the reproduction number of ${ }^{18}$ ${ }^{19} \mathrm{pH} 1 \mathrm{~N} 1$ because the transmission rate is higher in preschool and schoolchildren than ${ }^{19}$ ${ }^{20}$ in other age group individuals, in general. We consider a subgroup SIR model where ${ }^{20}$ ${ }^{21}$ the population is divided into $n_{a}$ age groups with different transmission dynamics. ${ }^{21}$ ${ }^{22}$ We denote the number of susceptible and infected individuals within the $i^{\text {th }}$ age ${ }^{22}$ ${ }^{23}$ group by $S_{i}$ and $I_{i}$, respectively. Let $\beta_{i j}$ refer to the transmission from the $j^{\text {th } 23}$ ${ }^{24}$ age group to the $i^{\text {th }}$ age group, and $\boldsymbol{\beta}=\left[\beta_{i j}\right]$ denote the transmission matrix, also ${ }^{24}$ ${ }^{25}$ known as Who-Acquires-Infection-From-Whom matrix. Putting these elements to- ${ }^{25}$ ${ }^{26}$ gether, we have the following system of differential equations. 26 $\begin{array}{ll}27 & 27\end{array}$ ${ }_{29}^{\prime}(t)=-\sum_{j=1}^{n_{a}} \beta_{i j} S_{i}(t) I_{j}(t)$ 30 $I_{31}^{\prime}(t)=\sum_{j=1} \beta_{i j} S_{i}(t) I_{j}(t)-\gamma_{i} I_{i}(t)$. 32 In a general structured model of the form (1) with $n_{a}$ distinct classes, $n_{a}^{2}$ trans- ${ }^{32}$ ${ }^{33}$ mission terms are required. However, one transmission term is available at most for ${ }^{33}$ 
${ }^{1}$ each class. The typical way to address this lack of specificity is to constrain the ${ }^{1}$ ${ }^{2}$ structure of the transmission matrix and/or to use prior knowledge of social mixing ${ }^{2}$ ${ }^{3}$ behavior. For an age-structured model, we assume that the transmission rates are ${ }^{3}$ ${ }^{4}$ proportional to the rates of social contact, which can be estimated from contact ${ }^{4}$ ${ }^{5}$ patterns. A large multi-country population-based survey conducted in Europe as a ${ }^{5}$ ${ }^{6}$ part of the POLYMOD [9] enables us to implement this approach. The transmis- ${ }^{6}$ ${ }^{7}$ sion is modeled as the product of the contact rate in the survey and an age-specific ${ }^{7}$ ${ }^{8}$ proportionality factor to account for characteristics related to susceptibility and ${ }^{8}$ ${ }^{9}$ infectiousness, which are not captured by contact rates. This leads to 9 10 $\begin{array}{ll}11 \\ 12\end{array} \quad \beta_{i j}= \begin{cases}q_{i} c_{i j} & i=j \\ \sigma c_{i j} & i \neq j\end{cases}$ 15 Based on the age-structured SIR model (1), the reproduction number can be ${ }_{15}$

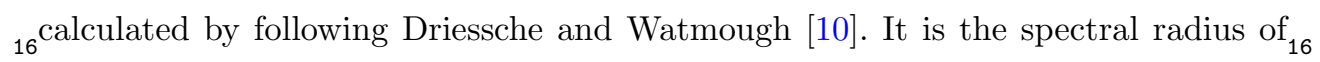
${ }_{17}$ the next generation matrix $M$ where account for the wave of the $\mathrm{pH} 1 \mathrm{~N} 1$ pandemic. We denote the number of susceptible 25 and infected individuals within the $i^{\text {th }}$ region by $S_{i}$ and $I_{i}$, respectively. Let $\beta_{i j}$ ${ }^{26}$ refer to the transmission from the $j^{\text {th }}$ subgroup to the $i^{\text {th }}$ subgroup and $\boldsymbol{\beta}=\left[\beta_{i j}\right]^{26}$ 27 denote the transmission matrix. In the same manner as the age-structured model, 28 we have the following system of differential equations. 
1 We assume that transmission rates between distinct regions in the region- ${ }^{1}$ ${ }^{2}$ structured model can be expressed as the frequency of transportations multiplied ${ }^{2}$ ${ }^{3}$ by a region-specific proportionality factor. The transportation information was ex- ${ }^{3}$ ${ }^{4}$ tracted from the highway portal site and Kakao map for number of buses and ${ }^{4}$ ${ }^{5}$ highway traffic, respectively $[11,12]$. Let the number of buses and highway traffic ${ }^{5}$ ${ }^{6}$ from region $j$ to region $i$ be denoted by $w_{i, j}$ and $W_{i, j}$, respectively. Note that $w^{6}$ ${ }^{7}$ is symmetric because the bus route is circular, although $W$ is not necessarily. The ${ }^{7}$ 
${ }^{1}$ technique of the least squares method. In general, parameter estimation is conducted ${ }^{1}$ ${ }^{2}$ by minimizing the cost function, which measures the difference between the model ${ }^{2}$ ${ }^{3}$ prediction and observation. Let $\boldsymbol{\theta}$ be the parameter set and time points $t_{j}\left(j={ }^{3}\right.$ $\left.{ }^{4} 1, \ldots, N\right)$ are uniformly distributed with daily time step. The data vector $\boldsymbol{y}_{j}\left(j={ }^{4}\right.$ $\left.{ }^{5} 1, \ldots, N\right)$ denotes the number of cases at time $t_{j}$. It is a scalar for basic SIR model, ${ }^{5}$ ${ }^{6}$ but it is a vector structured by age and region for age-stratified and region-stratified ${ }^{6}$ ${ }^{7}$ models, respectively. For example, the $\boldsymbol{y}_{j}$ is a column vector of length 15 for the ${ }^{7}$ ${ }^{8}$ age-structured model. We recast the mathematical model as 8 $\boldsymbol{x}^{\prime}(t)=\boldsymbol{g}(t, \boldsymbol{x}(t), \boldsymbol{\theta})$, ${ }_{16}$ surement error $\varepsilon_{j} \sim \mathcal{N}\left(0, a^{2}\right)$. The least squares estimator can be obtained by 16 ${ }_{17}$ minimizing the following cost function over the given parameter space $\Omega_{\theta}[13]$ : ${ }_{17}$

18

19

20 21 and region-structured model, are

22

$23 \quad \boldsymbol{\theta}_{\text {basic }}=\{\beta, \gamma\}$ and initial values of $S$ and $I, \quad 23$

$24 \boldsymbol{\theta}_{\text {age }}=\left\{q_{i}, \sigma, \gamma_{i}\right.$ for $\left.i=1,2, \cdots, n_{a}\right\}$ and initial values of $S_{i}$ and $I_{i}, \quad 24$

25

26

${ }_{27}$ where $n_{a}$ and $n_{r}$ denote the number of age groups and regions, respectively.

\section{${ }^{28}$ Results}

${ }^{29}$ Time-dependent reproduction number

${ }^{30}$ We illustrate the proposed methodology and investigate its performance by applying ${ }^{30}$ ${ }^{31}$ it to 2009 incidence data of pH1N1 in South Korea. This section presents the results ${ }^{31}$

${ }^{32}$ of the estimation obtained by applying the least squares method to basic SIR, ${ }^{32}$ ${ }^{33}$ age- and region-structured models. In each experiment, data with different time ${ }^{33}$ 
${ }^{1}$ periods by varying the terminal time are tested to determine the earliest stage of ${ }^{1}$

${ }^{2}$ the epidemic sufficient to provide a reasonable estimation. Figure 3 displays the ${ }^{2}$

${ }^{3}$ predicted incidence based on the basic SIR model compared with the observed ${ }^{3}$

${ }^{4}$ data. Predictions using data only during the initial growth phase cannot effectively ${ }^{4}$

${ }^{5}$ exhibit the dynamics and substantially overestimate the spread of the infection. ${ }^{5}$

${ }^{6}$ The results of simulation improved after the peak of the epidemic, and the wave ${ }^{6}$

${ }^{7}$ is roughly generated at a later stage. However, the simple SIR model does not ${ }^{7}$ ${ }^{8}$ provide a reasonable estimation of parameters. The estimated values of $\gamma$ and $R_{0}{ }^{8}$

${ }^{9}$ demonstrate a large variation and remains outside of the feasible range for the in- ${ }^{9}$

${ }^{10}$ fluenza, regardless of the time period for data in figure 6 . The plausible reason for ${ }^{10}$

${ }^{11}$ this involves the model assumptions that are too simple to capture the underlying ${ }^{11}$

${ }^{12}$ mechanisms. 12

${ }^{13}$ For the age-structured SIR model (1), the total population is split into 14 sub- $^{13}$

${ }^{14}$ groups of 5 -year age bands and one with 70 years and older (i.e., $0-4,5-9, \ldots, 65-69{ }^{14}$

${ }^{15} 70^{+}$). This incorporates a heterogeneous population into the model in order to re- ${ }^{15}$

${ }^{16}$ flect different transmission rates in each age group. In figure 4 , the outbreaks are ${ }^{16}$

${ }^{17}$ simulated using data during various time periods in the same manner as mentioned ${ }^{17}$

${ }^{18}$ above. Results of both the models show similar trends as long as the terminal time is ${ }^{18}$

19 earlier than mid-November when the gentle growth begins during the decline stage 19

${ }^{20}$ Additionally, as the growth begins to decline, the age-structured SIR model fits ${ }^{20}$

${ }^{21}$ the incidence data better than the basic SIR model. In figure 6 , the reproduction ${ }^{21}$

${ }^{22}$ number starts increasing in early October, peaks at 2.5 on October 17 , and then ${ }^{22}$

${ }^{23}$ decreases to unity at the end of October. Real-time estimation demonstrated that ${ }^{23}$

24 . 24

the effective reproduction number rose sharply during mid-October when the num-

${ }^{25}$ ber of patients increased dramatically. The reproduction number fell below unity at ${ }^{25}$

${ }^{26}$ the end of October and stayed lower than unity indicating that the epidemic starts ${ }^{26}$

${ }^{27}$ decreasing, which is consistent with the incidence data. In the age-stratified model, ${ }^{27}$

${ }^{28}$ heterogeneity was incorporated by WAIFW matrix where the transmission was as- ${ }^{28}$

${ }^{29}$ sumed to be proportional to the contacts. The effective contacts were measured by ${ }^{29}$

${ }^{30}$ POLYMOD contact survey, which showed a clear evidence for an age-dependency ${ }^{30}$

${ }^{31}$ in contact patterns. Taking heterogeneous mixing into the model enabled better ${ }^{31}$

${ }^{32}$ description of the dynamics, because the trend in behavior was consistent with the ${ }^{32}$

${ }^{33}$ demographic characteristics of cases (as shown in Table 1). Estimated parameters ${ }^{33}$ 
${ }^{1}$ are possible indicators to determine the feasibility of models. Incorporation of the ${ }^{1}$

${ }^{2}$ age structure allows for robust estimation of parameters, while the basic SIR model ${ }^{2}$ ${ }^{3}$ provides estimated values beyond the reasonable range with severe fluctuation in ${ }^{3}$ ${ }^{4}$ figure 6 . Table 2 summarizes the parameter estimates using three different models. ${ }^{4}$ ${ }^{5}$ The estimated duration of infectious period using age-structured model is around ${ }^{5}$ ${ }^{6} 3.8$. The reproduction number was estimated to be 1.6 which is similar to those ${ }^{6}$ ${ }^{7}$ obtained in Mexico, the United States, New Zealand, Peru, and Chile [2, 14-17]. ${ }^{7}$

8 The general characteristics of regional difference led us to consider a second type ${ }^{8}$ ${ }^{9}$ of heterogeneity in the model. The nation is split into 252 in the region-structured ${ }^{9}$ ${ }^{10}$ model (2), where the transmission rates are implemented based on transportation ${ }^{10}$ ${ }^{11}$ patterns. Figure 5 compares the predicted cases based on the region-structured $\mathrm{SIR}^{11}$ ${ }^{12}$ model with the observed data over the course of the epidemic. As it was discussed ${ }^{12}$ ${ }^{13}$ in the previous experiment, it is not earlier than the epidemic peak for estimation ${ }^{13}$ ${ }^{14}$ to start adjusting to outbreak data. Since this outbreak, the incidence data is well ${ }^{14}$ ${ }^{15}$ described in the form of the characteristic exponential rise, turnover, and decline ${ }^{15}$ ${ }^{16}$ pattern predicted by the process model. The estimated duration of infectious pe- ${ }^{16}$ ${ }^{17}$ riod using region-structured model is around 2.1 and the reproduction number was ${ }^{17}$ ${ }^{18}$ estimated to be 1.4 (Table 2). The time-dependent effective reproduction number ${ }^{18}$

${ }^{19}$ is also illustrated in figure 6 , which demonstrates a pattern similar to that obtained ${ }^{19}$ ${ }^{20}$ using the age-structured SIR model.

${ }^{23}$ It is widely known that the transmission is considerably different among various ${ }^{23}$ ${ }^{24}$ age groups. We also observe from the pH1N1 epidemic data that the incidence rate ${ }^{24}$ ${ }^{25}$ is higher in children and students than in other age groups (Table 1). Estimates of ${ }^{25}$ ${ }^{26}$ the age-specific reproduction number help in clarifying the role of each age group in ${ }^{26}$ ${ }^{27}$ the transmission dynamics and in suggesting guidelines for effective targeting inter- ${ }^{27}$ ${ }^{28}$ vention strategies. The estimated age-specific reproduction numbers are displayed ${ }^{28}$ ${ }^{29}$ in figure 7 . The result is closely related to the cumulative incidence for each age ${ }^{29}$ ${ }^{30}$ group because it is often the contact rate within the same age group is higher than ${ }^{30}$ ${ }^{31}$ with other groups. 
${ }^{1}$ mated the region-specific reproduction number and observed that it is more than ${ }^{1}$

${ }^{2}$ two in some areas and less than one in the others (Figure 8 ). This is consistent ${ }^{2}$

${ }^{3}$ with regions having larger cumulative incidence with a similar argument regarding ${ }^{3}$

${ }^{4}$ contact patterns to age-specific cases. 4

5

626

${ }_{7}$ Discussion 7

${ }^{8}$ An estimation of reproduction numbers is crucial because it provides a measure of ${ }^{8}$

${ }^{9}$ the transmission potential when an infection is spreading throughout a population. ${ }^{9}$

${ }^{10}$ The reproductive numbers in the early phase of the pandemic have been estimated ${ }^{10}$

${ }^{11}$ in several countries with different settings, yielding median 1.46 and range 1.0-3.6 ${ }^{11}$ ${ }^{12}[18]$. Many of these studies focused on cases confirmed in the early stage of the ${ }^{12}$ ${ }^{13}$ pandemic. Because laboratory tests focused on severe cases and there are possible ${ }^{13}$ ${ }^{14}$ changes in laboratory testing and notification rates, the number of confirmed cases $^{14}$ ${ }^{15}$ does not necessarily represent the underlying epidemic. It also does not reflect the ${ }^{15}$ ${ }^{16}$ dynamics during the period of the highest level of activity, which is the winter in ${ }^{16}$ ${ }^{17}$ temperate climates. Some studies used the number of cases from sentinel surveillance ${ }^{17}$ ${ }^{18}$ that is much less than the actual number of influenza patients. It is necessary to ${ }^{18}$ ${ }^{19}$ estimate the reproductive number using the number of all the patients throughout ${ }^{19}$ ${ }^{20} \mathrm{a}$ pandemic, including the period with the highest level of activity. In this study, ${ }^{20}$ ${ }^{21}$ the reproductive number of was estimated based on the national data of incidence ${ }^{21}$ ${ }^{22}$ deduced from antiviral agent prescription in South Korea during the pandemic. ${ }^{22}$ ${ }^{23}$ We discussed parameter estimation methodologies based on deterministic $\mathrm{SIR}^{23}$ ${ }^{24}$ models incorporating population heterogeneity. Age-structured and region-structured ${ }^{24}$ ${ }^{25}$ models were employed to describe the underlying epidemic process. The proposed ${ }^{25}$ ${ }^{26}$ mechanisms were applied to influenza A(H1N1)pdm09 in South Korea to compute ${ }^{26}$ ${ }^{27}$ the time-dependent effective reproduction numbers. We found that the introduc- ${ }^{27}$ ${ }^{28}$ tion of heterogeneity into the population and sufficient data to represent general ${ }^{28}$ ${ }^{29}$ characteristics of dynamics are essential to the robust estimation of parameters. ${ }^{29}$ ${ }^{30}$ Real-time estimation showed that the reproduction number started increasing in ${ }^{30}$ ${ }^{31}$ early October, peaked at 2.5 on October 17 , and then decreased to unity at the ${ }^{31}$ ${ }^{32}$ end of October. The effective number rose sharply during the mid-October when ${ }^{32}$ ${ }^{33}$ the number of patients increased dramatically. The reproduction number fell below ${ }^{33}$ 
${ }^{1}$ unity at the end of October and remained lower that unity, indicating that the ${ }^{1}$

${ }^{2}$ epidemic starts decreasing, which is consistent with the incidence data. ${ }^{2}$

${ }_{4}$ Subsequently, age-specific and region-specific basic reproduction numbers were ${ }_{4}$ ${ }_{5}$ estimated to account for the differences of incidence. We observe from the $\mathrm{pH}_{1} \mathrm{~N}_{5}$ ${ }_{6}$ epidemic data that the incidence rate is higher in children and students than in other ${ }_{6}$ ${ }_{7}$ age groups. The estimated age-specific reproduction numbers agree with the cumu- ${ }_{7}$ ${ }_{8}$ lative incidence for each age group because the mixing is assortative. The incidence ${ }_{8}$ ${ }_{9}$ rate is higher in urban areas than in rural areas, highest in the national capital and ${ }_{9}$ ${ }_{10}$ in the south-eastern region. We estimated the region-specific reproduction number ${ }_{10}$ ${ }_{11}$ whose trend is similar to the number of cases in each region. Estimates of the age- ${ }_{11}$ ${ }_{12}$ Specific and region-specific reproduction number help to predict the transmission ${ }_{12}$ ${ }_{13}$ dynamics, and to suggest guidelines for effective targeting intervention strategies. ${ }_{13}$

14 This study has both limitations and strengths. The first limitation is that the ${ }^{15}$ number of cases is estimated from the amount of prescribed antiviral agents as- ${ }^{15}$ ${ }^{16}$ suming the time lag between symptom onset and antiviral agent prescription, the ${ }^{16}$ ${ }^{17}$ proportion of prescription and the proportion of pH1N1 confirmation among $\mathrm{AFRI}^{17}$ ${ }^{18}$ patients. The effective contacts were employed from POLYMOD contact survey ${ }^{18}$ ${ }^{19}$ which possibly yields discrepancy in mixing pattern of Korea. The structured mod- ${ }^{19}$ ${ }^{20}$ els may not be enough to account for some underlying mechanism of dynamics ${ }^{20}$ ${ }^{21}$ due to those factors. However, they provide feasible estimates of reproduction num- ${ }^{21}$ ${ }^{22}$ ber despite the limitations, which was not possible using simple SIR model. We ${ }^{22}$ ${ }^{23}$ will be able to improve the outcome as we gather more information, because addi- ${ }^{23}$ ${ }^{24}$ tional knowledge is required to achieve a better result. Another limitation is that ${ }^{24}$ ${ }^{25}$ vaccination is not considered in the model. However, the effect of vaccination on ${ }^{25}$ ${ }^{26}$ the transmission of $\mathrm{pH} 1 \mathrm{~N} 1$ may have been insignificant because the vaccination for ${ }^{26}$ ${ }^{27}$ general group was initiated in January 2010. On the contrary, the present research ${ }^{27}$ ${ }^{28}$ has its strengths compared to previous studies. The reproduction number was esti- ${ }^{28}$ ${ }^{29}$ mated based on the national level antiviral agent prescription data in South Korea ${ }^{29}$ ${ }^{30}$ throughout the pandemic including the period of the highest level of activity. The 30 ${ }^{31}$ real-time estimation incorporating population structures can be used to predict the ${ }^{31}$ ${ }^{32}$ disease dynamics, thereby providing guidelines for the optimal implementation of ${ }^{32}$ 33 preventive measures, such as school closing and distribution of antiviral agents. 


\section{${ }^{1}$ Appendix}

${ }^{2}$ The reproduction number for the age-structured SIR model (1), can be calculated ${ }^{2}$

${ }^{3}$ following the approach of Driessche and Watmough [10]. Let $\mathcal{F}_{i}$ be the new infections ${ }^{3}$

${ }^{4}$ and $\mathcal{V}_{i}$ be the transitions of $i^{t h}$ compartment, then

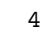

5

${ }_{7} \quad \mathcal{F}_{i}=S_{i}\left(q_{i} c_{i i} I_{i}+\sum_{j \neq i} \sigma c_{i j} I_{j}\right)$

sand

9

$$
\mathcal{V}_{i}=\gamma I_{i}
$$

10

${ }^{11}$ for $i=1, \cdots, n_{a}$.

12 Subsequently, the derivatives of $\mathcal{F}=\left[\mathcal{F}_{i}\right]$ and $\mathcal{V}=\left[\mathcal{V}_{i}\right]$ are 12

13

${ }_{15} \quad F=\left[\begin{array}{cccc}S_{1} q_{1} c_{11} & S_{1} \sigma c_{12} & \cdots & S_{1} \sigma c_{1 n_{a}} \\ S_{2} \sigma c_{21} & S_{1} q_{2} c_{22} & \cdots & S_{2} \sigma c_{2 n_{a}} \\ \vdots & \vdots & \ddots & \vdots \\ S_{n_{a}} \sigma c_{n_{a} 1} & S_{n_{a}} \sigma c_{n_{a} 2} & \cdots & S_{n_{a}} q_{n_{a}} c_{n_{a} n_{a}}\end{array}\right], \quad V=\left[\begin{array}{cccc}\gamma & 0 & \cdots & 0 \\ 0 & \gamma & \cdots & 0 \\ \vdots & \vdots & \ddots & \vdots \\ 0 & 0 & \cdots & \gamma\end{array}\right] \begin{gathered}14 \\ 16 \\ 17\end{gathered}$

${ }^{18}$ respectively, and the next generation matrix is

$$
F V^{-1}=\frac{1}{\gamma}\left[\begin{array}{cccc}
S_{1} q_{1} c_{11} & S_{1} \sigma c_{12} & \cdots & S_{1} \sigma c_{1 n_{a}} \\
S_{2} \sigma c_{21} & S_{1} q_{2} c_{22} & \cdots & S_{2} \sigma c_{2 n_{a}} \\
\vdots & \vdots & \ddots & \vdots \\
S_{n_{a}} \sigma c_{n_{a} 1} & S_{n_{a}} \sigma c_{n_{a} 2} & \cdots & S_{n_{a}} q_{n_{a}} c_{n_{a} n_{a}}
\end{array}\right] . \quad \begin{array}{r}
19 \\
20 \\
22 \\
23
\end{array}
$$

${ }^{24}$ Thus, the reproduction number is the spectral radius of $F V^{-1}$ and the age-specific ${ }^{24}$ ${ }^{25}$ reproduction number is the column sum of $F V^{-1}$ corresponding to the age of in- ${ }^{25}$ ${ }^{26}$ terest.

This study was approved by the Institutional Review Board (IRB) of Yonsei University Health System.

${ }^{31}$ Availability of data and materials 31

32 The dataset used and/or analysed during the current study were employed from the literature [8]. 32

${ }_{33}$ Competing interests 
${ }_{4} \mathrm{DH}$ and $\mathrm{J}$ designed the work, and $\mathrm{C}$ contributed to collect data. $\mathrm{Y}$ carried out data processing and numerical

${ }_{11}^{1}$ Department of Computational Science and Engineering, Yonsei University, 50, Yonsei-ro, 03722, Seoul, South

${ }^{11}$ Korea. ${ }^{2}$ Korea Centers for Disease Control and Prevention, 187, Osongsaengmyeong 2-ro, 28159, Cheongju-si,

12 South Korea. ${ }^{3}$ Department of Mathematics, Inha University, 100, Inha-ro, 22212, Incheon, South Korea. 12

${ }^{4}$ Department of Preventive Medicine and Public Health, Severance Hospital, Yonsei University College of Medicine,

${ }^{13} 50-1$, Yonsei-ro, 03722, Seoul, South Korea. ${ }^{5}$ Department of Mathematics, Yonsei University, 50, Yonsei-ro, $03722,{ }^{13}$ 14 Seoul, South Korea.

1. Cruz-Pacheco G, Duran L, Esteva L, Minzoni AA, López-Cervantes M, Panayotaros P, et al. Modelling of the

16 influenza $A(H 1 N 1)$ outbreak in Mexico City, April-May 2009, with control sanitary measures. Euro Surveill. 16 $17 \quad 2009 ; 14(26)$.

2. Fraser C, Donnelly CA, Cauchemez S, Hanage WP, Van Kerkhove MD, Hollingsworth TD, et al. Pandemic

18 potential of a strain of influenza A (H1N1): early findings. Science. 2009;324(5934):1557-61.

19 3. Nishiura H, Chowell G, Safan M, Castillo-Chavez C. Pros and cons of estimating the reproduction number from 19 early epidemic growth rate of influenza A (H1N1) 2009. Theor Biol Med Model. 2010;7(1).

20 4. Yang Y, Sugimoto JD, Halloran ME, Basta NE, Chao DL, Matrajt L, et al. The transmissibility and control of 20 pandemic influenza A (H1N1) virus. Science. 2009;326(5953):729-33.

21 5. Cowling BJ, Lau MSY, Ho LM, Chuang SK, Tsang T, Liu SH, et al. The effective reproduction number of 21

22 pandemic influenza: prospective estimation. Epidemiology. 2010;21(6):842-6.

6. World Health Organization. Influenza-like illness in the United States and Mexico, 24 April 2009; 2009.

23 Available from: https://www.who.int/csr/don/2009_04_24/en/. Accessed 4 Sep 2019.

24 7. Korea Centers for Disease Control and Prevention. Analysis of reported pandemic influenza (A/H1N1 2009) 24 virus infections in Korea - From April, 2009 through August, 2010. Public Health Weekly Report 2010a.

25 2010;3:637-42. 25

26 8. Lee DH. Estimation of the reproduction number of pandemic influenza A(H1N1) 2009 from national data of 26 antiviral agent prescription. Yonsei University, Seoul, South Korea; 2011.

27 9. Mossong J, Hens N, Jit M, Beutels P, Auranen K, Mikolajczyk R, et al. Social contacts and mixing patterns 27 28 relevant to the spread of infectious diseases. PLoS Med. 2008 03;5(3).

29 compartmental models of disease transmission. Math Biosci. 2002;180:29-48. 29

11. Kakao map. Kakao Corporation, Jeju-si, South Korea; 2017. Available from: https://map.kakao.com/.

$30 \quad$ Updated 25 February 2019; Accessed 15 March 2017.

31 12. Highway public data portal. Korea Expressway Corporation, Gimcheon-si, South Korea; c2015. Available from: 31 http://data.ex.co.kr/portal/fdwn/view?type=TCS\&num=39\&requestfrom=dataset\#. Accessed $15 \mathrm{March}$

322017.

33 13. Banks HT, Hu S, Thompson WC. Modeling and inverse problems in the presence of uncertainty. New York: 33 Chapman and Hall/CRC; 2014. 
14. Paine S, Mercer G, Kelly P, Bandaranayake D, Baker M, Huang Q, et al. Transmissibility of 2009 pandemic

influenza A (H1N1) in New Zealand: effective reproduction number and influence of age, ethnicity and 2 importations. Euro Surveill. 2010;15(24):19591.

315. Munayco C, Gomez J, Laguna-Torres V, Arrasco J, Kochel T, Fiestas V, et al. Epidemiological and 3

4 transmissibility analysis of influenza A (H1N1) v in a southern hemisphere setting: Peru. Euro Surveill. 2009;14(32):19299.

516. Yang Y, Sugimoto JD, Halloran ME, Basta NE, Chao DL, Matrajt L, et al. The transmissibility and control of 5 pandemic influenza A (H1N1) virus. Science. 2009;326(5953):729-733.

6 17. Pedroni E, Garcia M, Espinola V, Guerrero A, Gonzalez C, Olea A, et al. Outbreak of 2009 pandemic influenza ${ }^{6}$

7 A (H1N1), Los Lagos, Chile, April-June 2009. Euro Surveill. 2010;15(1):19456.

18. Biggerstaff M, Cauchemez S, Reed C, Gambhir M, Finelli L. Estimates of the reproduction number for seasonal,

8 pandemic, and zoonotic influenza: a systematic review of the literature. BMC Infect Dis. 2014 Sep;14:480. 8

${ }^{9}$ Figures

$10 \longrightarrow$

Figure 1 Daily antiviral agent prescription (dotted line) and incident patients (solid line) from September 1, 2009 to March 30, 2010. The incidence data used for model calibration was 12 imported from literature and refer to [8] for details. 4 .

$\begin{array}{lll}14 & 14 \\ 15 & \text { Figure 2 Cumulative incidence by region. } & 15\end{array}$

16

Figure 3 Comparison of pH1N1 incidence data with predictions of least squares method (LSM) using the basic SIR model (top and bottom left): The red dots show the number of new cases per day and the blue line presents the predicted number of cases. Terminal time of data used for 19 estimation is displayed by a black dotted vertical bar. In each figure, the end of time period was set at October 24, 2009, November 14, 2009, and March 30, 2010.

Figure 4 Comparison of pH1N1 incidence data with predictions of least squares method (LSM) 3 using age-structured SIR model (top and bottom left): The red dots show the number of new cases per day, and the blue line presents the predicted number of cases. Terminal time of data used for estimation is displayed by a black dotted vertical bar. In each figure, the end of time period was set at October 24, 2009, November 14, 2009, and March 30, 2010.

Figure 5 Comparison of pH1N1 incidence data with the predictions of least squares method (LSM) obtained using the region-structured SIR model (top and bottom left): The red dots show the number of new cases per day, and the blue line presents the predicted number of cases. The terminal time of data used for estimation is displayed by a black dotted vertical bar. In each figure, the end of time period was set at October 24, 2009, November 14, 2009, and March 30, 2010. 


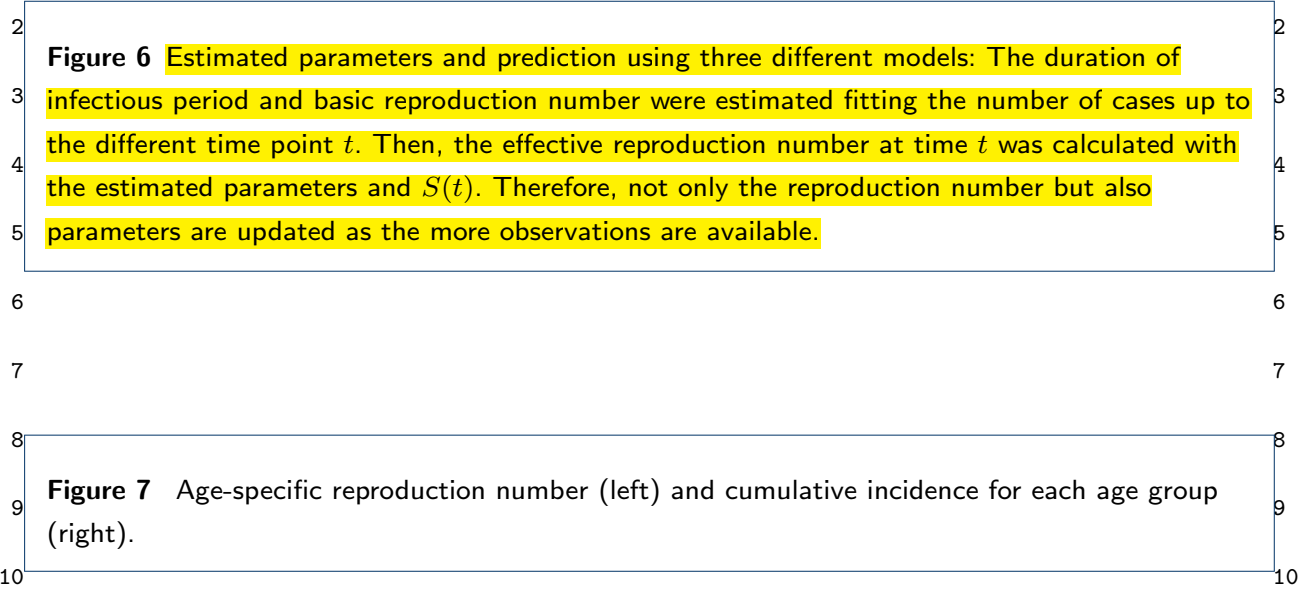

${ }_{17}$ Table 1 Cumulative incidence by age.

\begin{tabular}{cccc}
\hline Age & Population size & Total number of cases & Incidence rate(\%) \\
\hline $0-4$ & 2273634 & 917793 & 40 \\
$5-9$ & 2671496 & 1084094 & 41 \\
$10-14$ & 3284134 & 920937 & 28 \\
$15-19$ & 3368818 & 781238 & 23 \\
$20-24$ & 3153001 & 305403 & 10 \\
$25-29$ & 4001090 & 341940 & 9 \\
$30-34$ & 3856438 & 290354 & 8 \\
$35-39$ & 4466962 & 303670 & 7 \\
$40-44$ & 4244225 & 220743 & 5 \\
$45-49$ & 4321262 & 164735 & 4 \\
$50-54$ & 3761143 & 149067 & 4 \\
$55-59$ & 2611705 & 97571 & 4 \\
$60-64$ & 2117041 & 73540 & 3 \\
$65-69$ & 1870279 & 66033 & 4 \\
$70+$ & 3306607 & 100728 & 3 \\
\hline
\end{tabular}

30Table 2 Estimated parameters using three different models.

\begin{tabular}{cccc}
\hline & Basic SIR & Age-structured SIR & Region-structured SIR \\
\hline$R_{0}$ & 1.0722 & 1.6398 & 1.4487 \\
Infectious period & 0.5462 & 3.8539 & 2.1359 \\
\hline
\end{tabular}


Figures

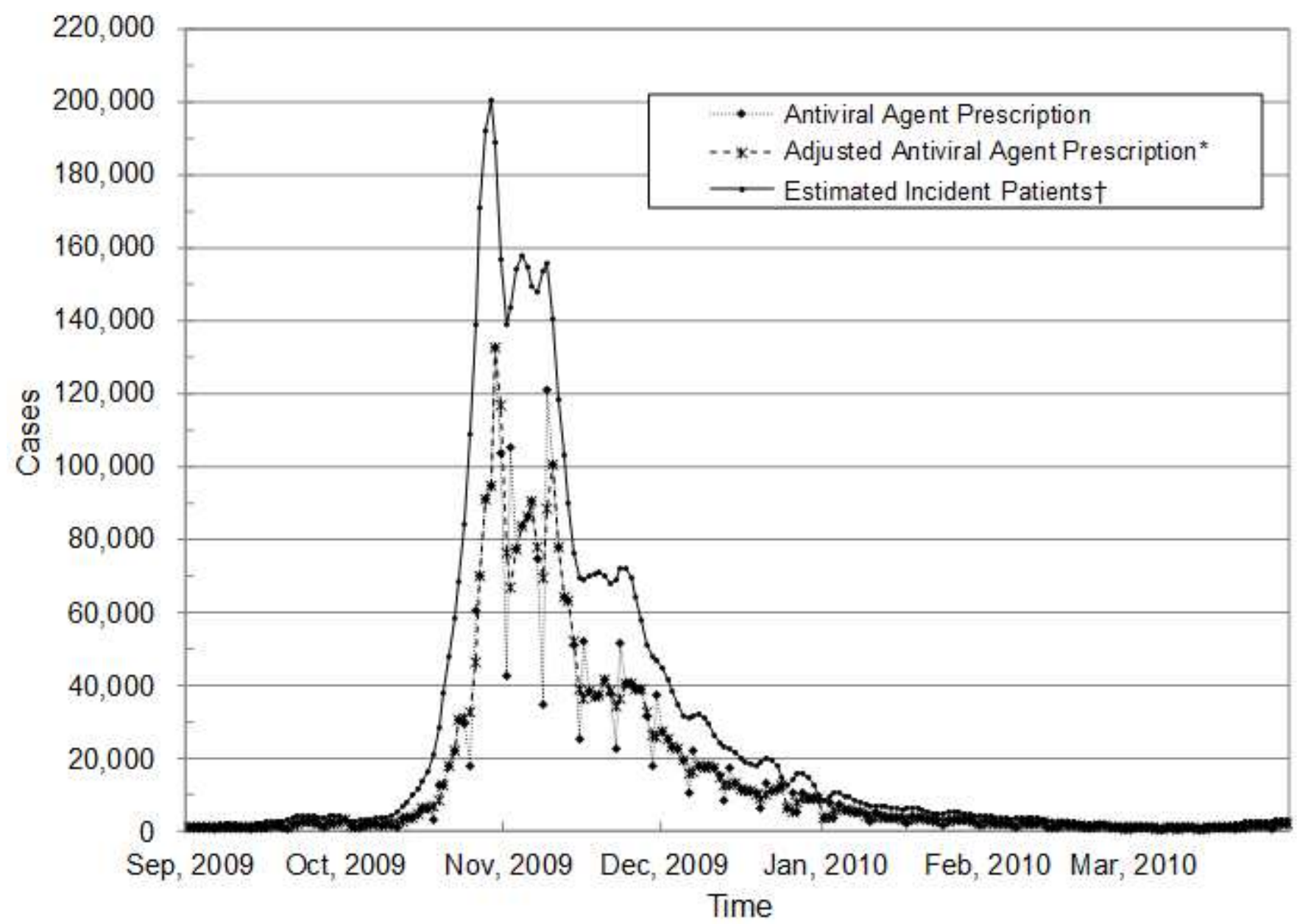

\section{Figure 1}

Daily antiviral agent prescription (dotted line) and incident patients (solid line) from September 1, 2009 to March 30, 2010. The incidence data used for model calibration was imported from literature and refer to [8] for details. 


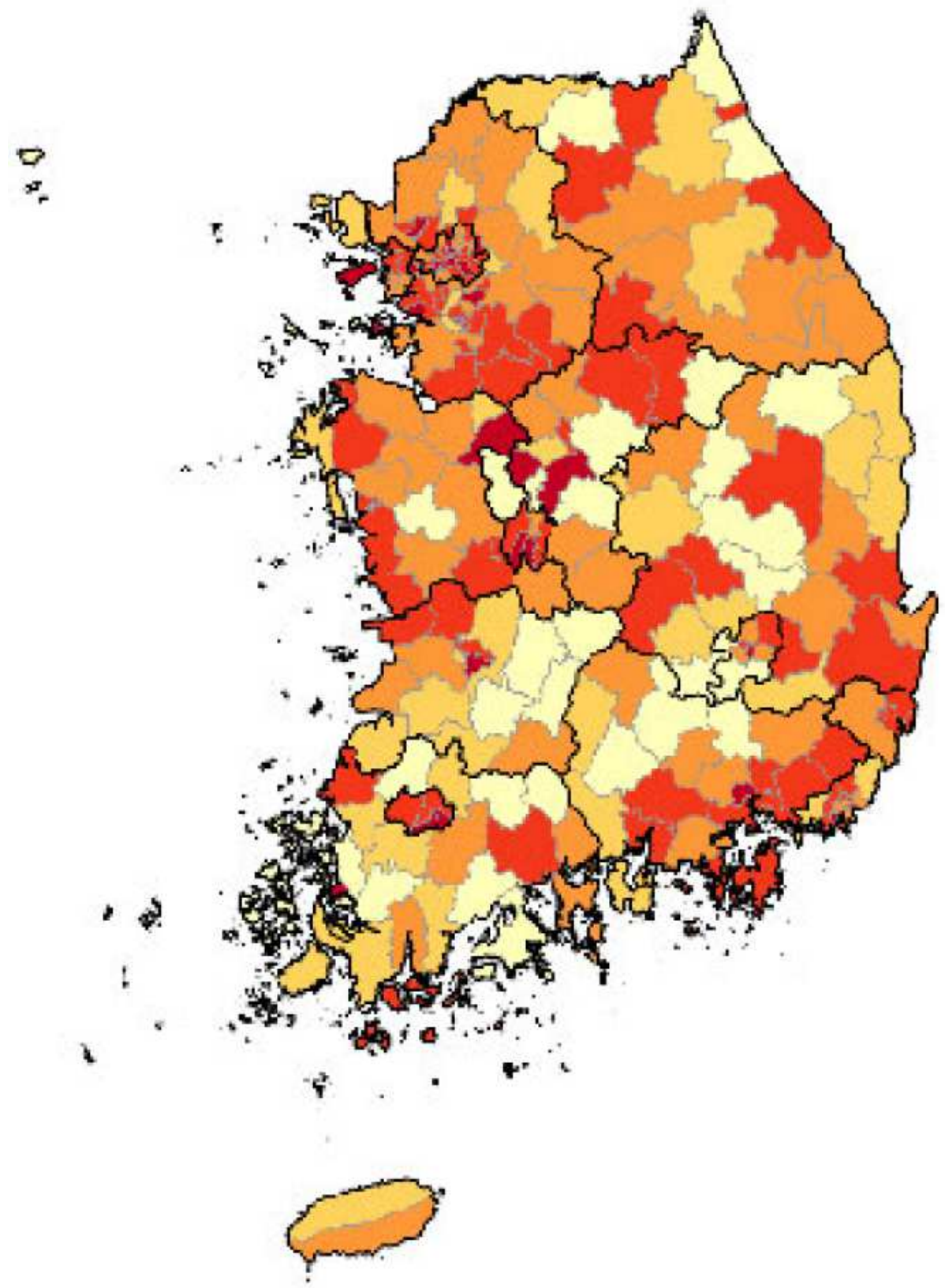

\section{Figure 2}

Cumulative incidence by region. Note: The designations employed and the presentation of the material on this map do not imply the expression of any opinion whatsoever on the part of Research Square concerning the legal status of any country, territory, city or area or of its authorities, or concerning the delimitation of its frontiers or boundaries. This map has been provided by the authors. 

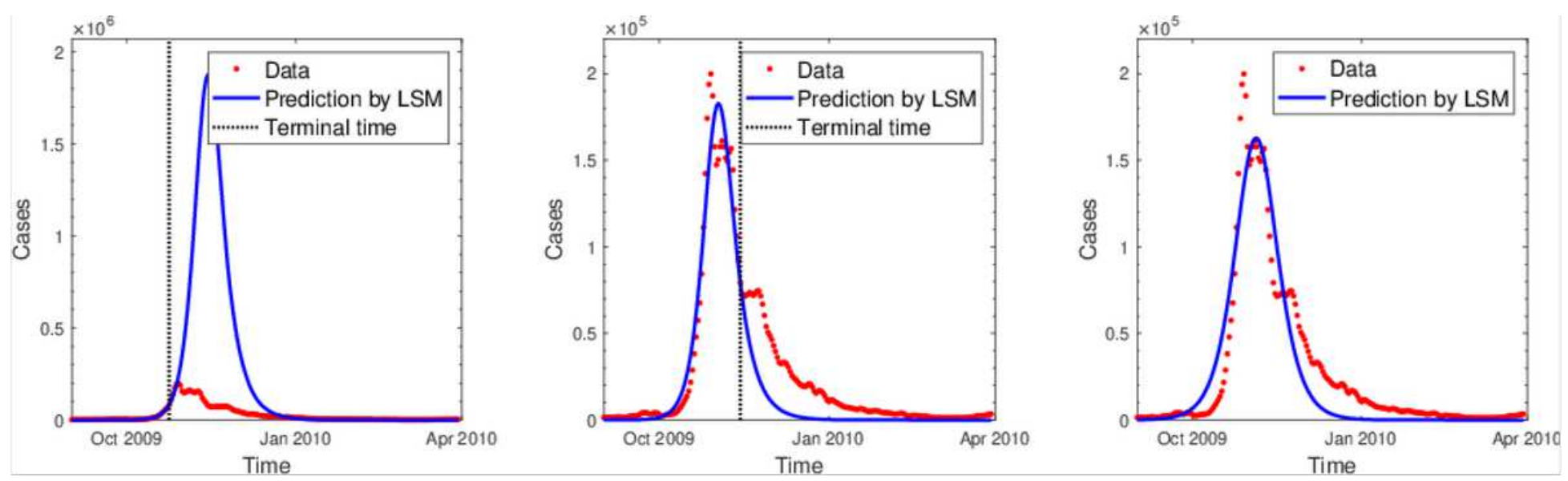

\section{Figure 3}

Comparison of pH1N1 incidence data with predictions of least squares method (LSM) using the basic SIR model (top and bottom left): The red dots show the number of new cases per day and the blue line presents the predicted number of cases. Terminal time of data used for estimation is displayed by a black dotted vertical bar. In each figure, the end of time period was set at October 24, 2009, November 14, 2009, and March 30, 2010.
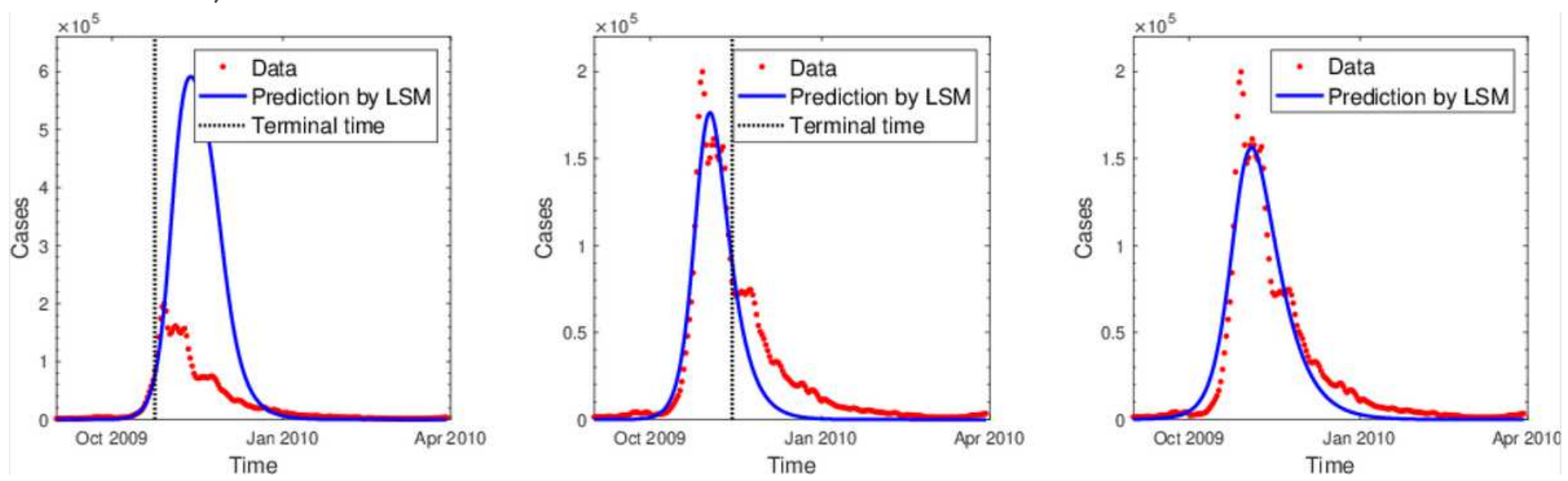

\section{Figure 4}

Comparison of pH1N1 incidence data with predictions of least squares method (LSM) using agestructured SIR model (top and bottom left): The red dots show the number of new cases per day, and the blue line presents the predicted number of cases. Terminal time of data used for estimation is displayed by a black dotted vertical bar. In each figure, the end of time period was set at October 24, 2009, November 14, 2009, and March 30, 2010. 

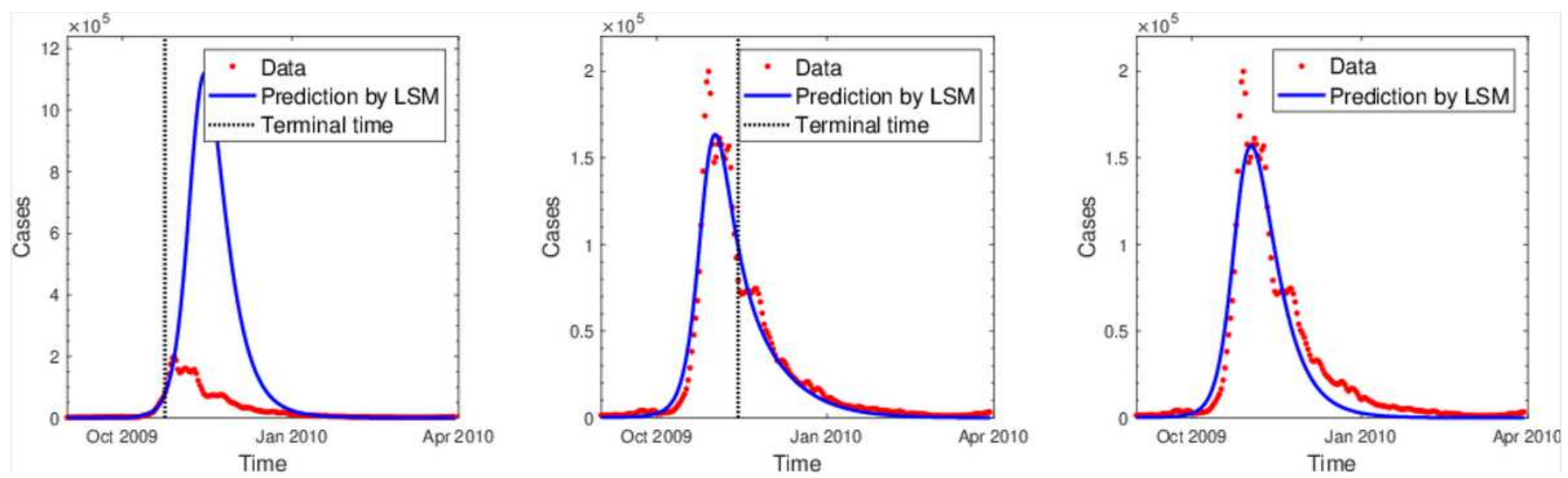

Figure 5

Comparison of pH1N1 incidence data with the predictions of least squares method (LSM) obtained using the region-structured SIR model (top and bottom left): The red dots show the number of new cases per day, and the blue line presents the predicted number of cases. The terminal time of data used for estimation is displayed by a black dotted vertical bar. In each figure, the end of time period was set at October 24, 2009, November 14, 2009, and March 30, 2010.
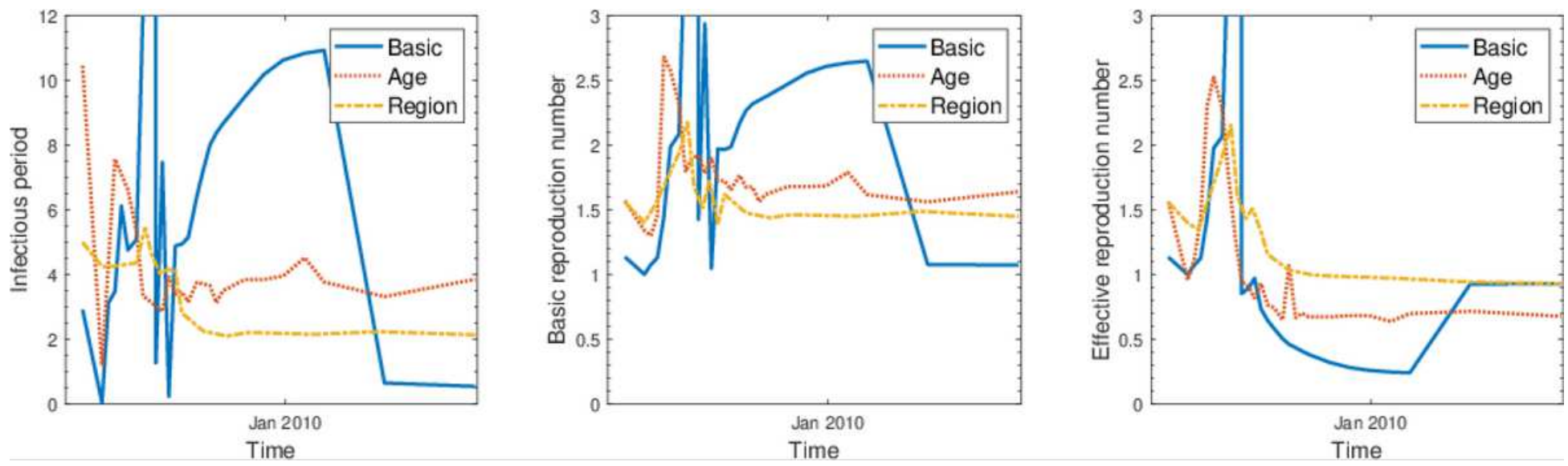

Figure 6

Estimated parameters and prediction using three different models: The duration of infectious period and basic reproduction number were estimated fitting the number of cases up to the different time point $t$. Then, the effective reproduction number at time $t$ was calculated with the estimated parameters and $S(t)$. Therefore, not only the reproduction number but also parameters are updated as the more observations are available. 


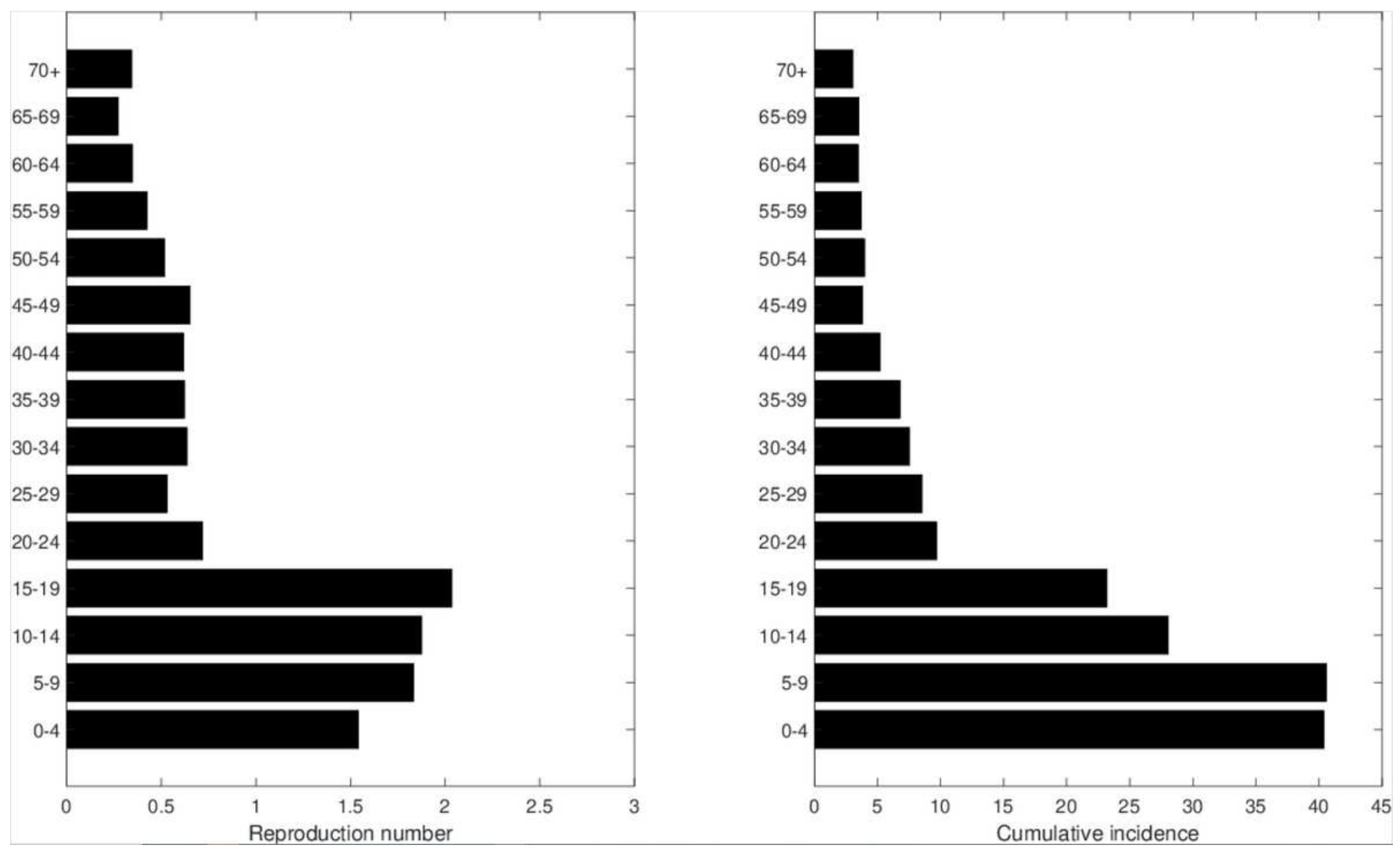

Figure 7

Age-specific reproduction number (left) and cumulative incidence for each age group (right). 

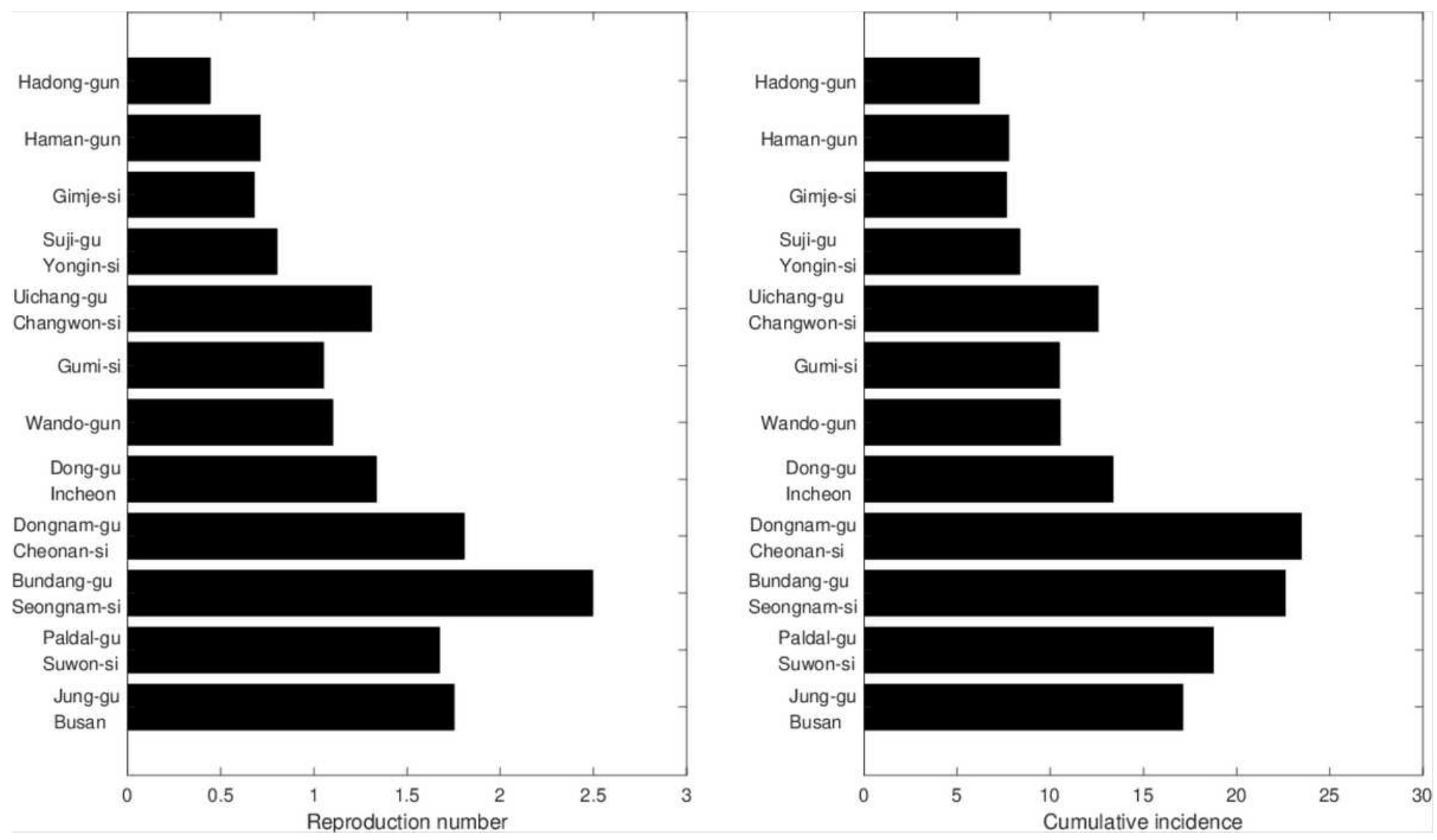

Figure 8

Region-specific reproduction number (left) and cumulative incidence for each region (right). 